Research letter

\title{
Development of the medical apparatus for Doppler-controlled treatment of hemorrhoids using laser coagulation
}

\author{
Nikolay A. Gryaznov ${ }^{1}$, Vyacheslav V. Kharlamov ${ }^{1}$, Sergey A. Nikitin ${ }^{1}$, Igor M. Cybin ${ }^{2}$, Konstantin Y. Senchik ${ }^{1}$, \\ Denis G. Shamarin ${ }^{1}$, Alina Y. Karseeva ${ }^{1}$, Galina S. Kireeva ${ }^{1,3}$ \\ ${ }^{1}$ Central Research Institute for Robotics and Technical Cybernetics, Saint-Petersburg, Russia \\ ${ }^{2}$ NPF «BIOSS», Saint-Petersburg, Russia \\ ${ }^{3}$ N.N. Petrov National Medical Research Center of Oncology, Saint-Petersburg, Russia
}

Received 3 July 2017, Accepted 23 September 2017

(C) 2017, Gryaznov N.A., Kharlamov V.V., Nikitin S.A., Cybin I.M., Senchik K.Y., Shamarin D.G., Karseeva A.Y., Kireeva G.S.

C 2017, Russian Open Medical Journal

\begin{abstract}
In the article authors present the concept of the surgical manipulator delivering laser radiation to the target area. For the implementation of Doppler-controlled treatment of hemorrhoids by laser coagulation, an important circumstance is the necessity to realize by means of the dopplerograph the possibility of controlling the efficiency of coagulation of the pathological vessel immediately after the impact, which will create the prerequisites for the formation of a program cycle with the inclusion of control elements by feedback. In this regard, the structure of the laser medical apparatus is developed, which allows determining the location, size and flow of arterial and venous vessels, performing laser coagulation of pathologically damaged sites under constant Doppler control. The developed adaptive power management system includes several functional units for the processing of the data from ultrasound scanner and the Doppler. Medical apparatus for minimally invasive treatment of hemorrhoids based on the laser coagulator and ultrasound Doppler will allow performing surgeries in automated and half-automated modes. The suggested medical apparatus helps to determine pathological vessels, choose the required radiation mode and provides vessel obliteration. Successful realization of constructive combination of real-time diagnostics and surgical manipulation with a laser can result in a unique minimally invasive solution to treat hemorrhoids that currently doesn't have analogues.
\end{abstract}

Keywords: hemorrhoids, laser coagulation, Doppler, minimally invasive surgery.

Cite as Gryaznov NA, Kharlamov VV, Nikitin SA, Cybin IM, Senchik KY, Shamarin DG, Karseeva AY, Kireeva GS. Development of the medical apparatus for Doppler-controlled treatment of hemorrhoids using laser coagulation. Russian Open Medical Journal 2017; 6: e0409.

Correspondence to Galina S. Kireeva. Address: Tikhoretsky prospect, 21, Saint-Petersburg, 194064, Russia. Phone: +7 (812) $439-95-55$, ext. 4315.

E-mail: galinakireyeva@mail.ru

\section{Introduction}

Hemorrhoids is one of the most common proctologic diseases. It is diagnosed in about $10 \%$ of adults, including almost $50 \%$ of men at the age of 60 and older [1]. At present, different methods of treating hemorrhoids exist, and they can be divided into conservative, minimally invasive and surgical. In cases of early stages of the disease conservative medical therapy and minimally invasive approaches, including laser coagulation, are successfully utilized [2-4]. Laser coagulation have several advantages including lack of pain, very short recovery period, as well as no need for hospitalization of the patient, since it is considered as an outpatient procedure [5].

Not so long ago the only radical treatment option for patients with hemorrhoids was surgery [2]. Nowadays several minimally invasive procedures are available along with the surgery. All these procedures are based on interruption of blood inflow to venous vessels of the rectum which leads to reduction of hemorrhoidal nodes $[3,4]$. Laser coagulation is one of the modern minimally invasive techniques for the treatment of hemorrhoids [5]. The main advantage of a laser surgical apparatus over other medical devices (ultrasonic dissectors, plasma coagulators) is the opportunity for very precise concentration of laser energy in the area of less than $0.1 \mathrm{~mm}$, and for selective thermodestruction, coagulation and vaporization of the tissues. During the procedure where medical lasers are used the vessels supplying hemorrhoids in sub-mucosal layer are sclerosed and obliterated. Currently more and more often laser coagulation is being chosen for the treatment of hemorrhoids due to several positive factors: it is an outpatient procedure, low recurrence rate, short postoperative period, it is a relatively simple and painless procedure.

During the laser coagulation session, it is important not only to diagnose pathological vessels, but also to control the course of the operation visually. The area of medicine where diagnostics and treatment are combined is called theranostics. Such combination improves efficacy of the treatment as whole $[6,7]$.

Minimally invasive laser procedure to treat hemorrhoids (HeLP) has been described [8]. Briefly, Doppler ultrasound sensor is inserted in the special cavity of the anoscope, so that it locates 
the artery to be coagulated. Anoscope position is fixed. Then the sensor is removed and, with the anoscope position fixed, a fiber optic cable with a special tip is inserted into the same cavity, and percutaneous coagulation of the artery is performed. However such technique doesn't provide either visual control during the procedure or observation of the efficacy of laser radiation in tissues.

Further development of laser surgery to treat hemorrhoids will be in achieving synergistic effect of combining modern diagnostic ultrasound module and a laser in one portable apparatus. Such apparatus would provide control of the procedure in real time.

The aim of our work was to develop a model of the device, which would combine two major functions (diagnostics and treatment of hemorrhoids) in time and space, allowing to achieve a new quality of the surgery.

\section{Material and Methods}

A novel medical apparatus (MA) operating in automated or half-automated regimens is required to treat hemorrhoids by laser coagulation of the vessels under control of the ultrasound dopplerography. An important factor is the need to implement the ability to control the efficiency of coagulation of the pathological vessel with the help of Doppler, immediately after the impact. That will create the prerequisites for the formation of a procedure cycle with the inclusion of control elements by feedback. The developed MA should include the following options:

i) search for the location of arterial and venous vessels,

ii) laser coagulation of pathologic vessesls,

iii) coagulation control.

Proceeding from the functional purpose of MA it is rational to build its structure based on its functional features. So the MA consists of the diagnostics unit, laser unit and control system. The structural scheme of the MA is presented in Figure 1. Doppler sensors 11 and ultrasound sensors 12 are constructively integrated with a fiber optic tool 14 into a surgical manipulator 19 . Construction of the surgical manipulator should ensure its compatibility with medical proctoscopes.

MA 1 performs as follows. Information from the Doppler sensors 11 and ultrasound sensors 12 goes to the preprocessing blocks of the Doppler control system 17 and US control system 18, then goes to the interface module 9 of the MA control system and is presented on the display 15. Data processing of Doppler and ultrasound sensors is performed in the calculator 10 . The calculator 10 forms control commands for the laser unit (LU) 3 which then go to the LU control system 8 . LU control system 8 sets the operating mode of the laser emitter 7. LU power and cooling system 13 maintains the required energy and temperature regimens for the laser emitter 7 .

Configuration of the surgical manipulator, allowing delivery of laser radiation into the targeted zone, can look as follows (Figure 2). The designs of the sketch models differ from each other in the shape of the fiber guide (A2, B2, B2) of the light guide (A1, $B 1, B 1)$ and the kinematic scheme of the mechanism by which the guide is fed to the target area. Surgical intervention is made by inserting a fiber into a body along its movement direction (A3, B3, B3) to the center of the target area 5 . When the required position is achieved laser radiation of the affected vessel area is performed.

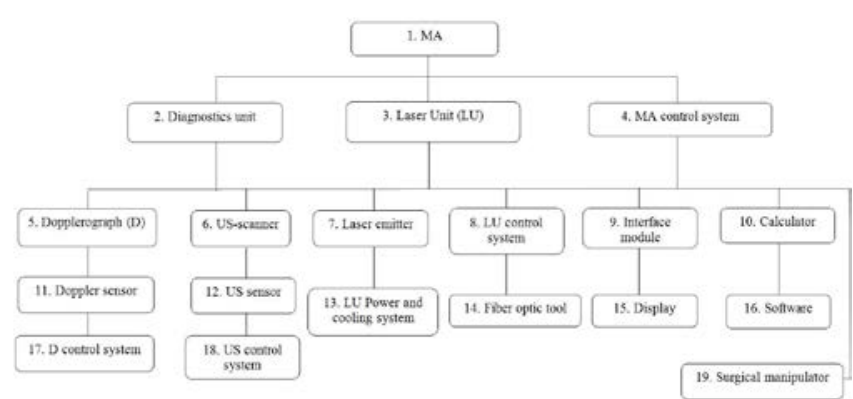

Figure 1. Structural scheme of the developed medical apparatus
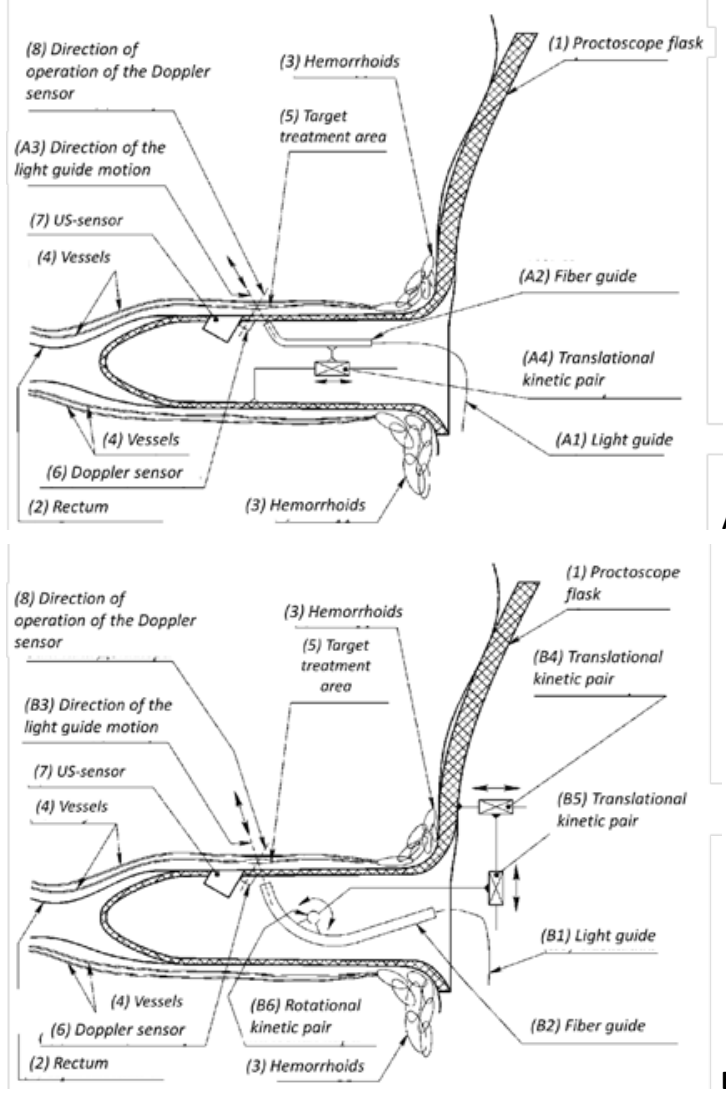

A

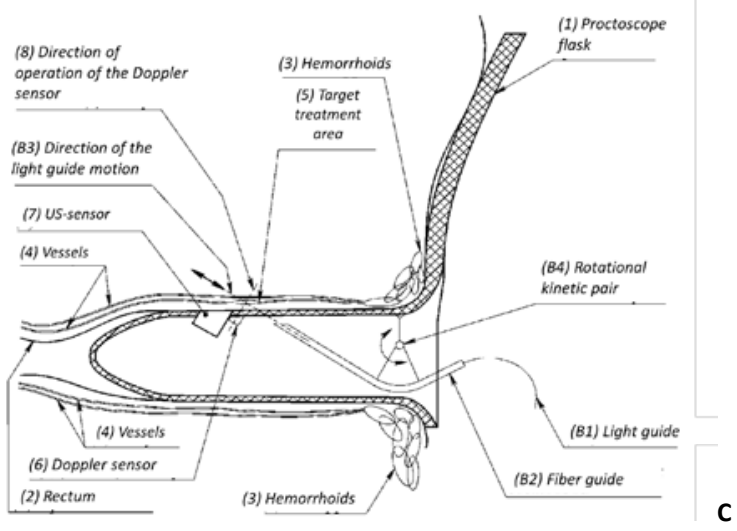

Figure 2. Variations of mechanical manipulator of the developed model: A) design with a L-shaped fiber guide and a mechanism with one translational kinematic pair; B) design with a L-shaped fiber guide and a mechanism with two translational and one rotational kinematic pair; C) design with a straight fiber guide and a mechanism with one rotational kinematic pair. US, ultrasound. 


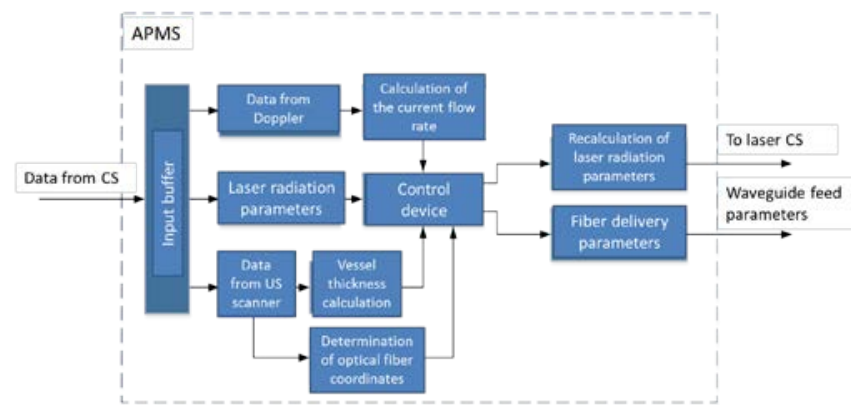

Figure 3. Functional scheme of the adaptive power management system (APMS). CS, control system; US, ultrasound.

Parameters of the laser radiation depend on characteristics of the irradiated vessels. Therefore adaptive power management system (APMS) of the laser radiation is required (Figure 3). APMS adapts the parameters of the output radiation, analyzes the data coming from the ultrasound scanner and the Doppler.

Data from the Doppler, ultrasound scanner and laser radiation parameters got to the input buffer of APMS where they undergo primary processing. Data obtained from the Doppler, go from the input buffer to the FU of calculation of the flow rate in the vessel. Data determining laser radiation parameters go to the FU of laser parameters processing where current laser parameters in the impact point are determined. Vessel thickness in the impact point and current light guide coordinates are determined according to the data from the ultrasound scanner. Processed data from the FU go to the control device. Based on the obtained information and the set parameters, the control device recalculates and adapts the control actions to the fiber delivery module and the laser control system. New adapted laser radiation parameters and its target coordinates go to the laser control system and to the fiber delivery system respectively.

\section{Results and Discussion}

Laser coagulation procedure to treat hemorrhoids using novel MA described previously will performed as follows. Firstly a proctoscope will be introduced into the anus. An ultrasound scanner will be used to determine pathological vascular discharges, the sizes and course of the vessels. The entire procedure is performed with continuous ultrasound Doppler control. After finding the vessel, the optimal regimen of laser radiation will be chosen automatically based on the obtained data about the size of the vessel, blood flow rate and severity of the pathological process. Then laser coagulation of the vessel will be performed, i.e. light guide will be led to the chosen vessel by means of the surgical manipulator (light guide position is controlled with the ultrasound scanner). During the procedure blood inside the vessel coagulates, and the vessel collapses. It is the complete gluing of the vessel that is the achievable goal and is controlled by the Doppler sensor. In fact, the laser beam affects the vessel wall indirectly. The maximum absorption of laser energy is due to the blood contained in the vessel. Under the influence of a light pulse, vapor bubbles form in the blood, and the thermal effect on the vein wall is due to its contact with these bubbles. This leads to direct damage to the endothelium and coagulation of the proteins in the subendothelial layers.

Clinical criteria of coagulation efficiency are:

i) darkening - the vessel becomes dark purple or gray (with thrombosis or with a vessel rupture);

ii) blanching of the vessel, "filiform" appearance;

iii) sometimes visual disappearance of the vessel (with its spasm).

After the impact of laser radiation on the vessel, the vessel condition is evaluated according to the clinical criteria presented above, and the decision about the need for repeated irradiation is made.

Data from the ultrasound Doppler processed in APSM is used to control time and temperature of laser irradiation, and the required impact area.

Use of the new MA could simplify performance of minimally invasive surgeries to treat hemorrhoids and improve their accuracy.

\section{Conclusion and Future Directions}

Successful realization of constructive combination of real-time diagnostics and surgical manipulation with a laser can result in a unique minimally invasive solution to treat hemorrhoids that currently doesn't have analogues. Also this idea could be the basis for construction of medical devices for minimally invasive surgeries in other areas of medicine, for example, for the treatment of vascular and oncological diseases.

\section{Acknowledgments}

This article was prepared with financial support from the Ministry of Education and Science of Russian Federation for the research under the Agreement of 03.10.2016, grant №14.578.21.0207 (unique identifier RFMEFI57816X0207) for the implementation of the federal target program "Research and development on priority directions of scientifictechnological complex of Russia for 2014-2020 years".

\section{Conflict of interest}

Authors declare they have no conflict of interest.

\section{References}

1. Guide to primary health care. A.A. Baranov, I.N. Denisova, A.G Chuchalina, eds. Moscow, Russia: Geotar-Media, 2006; 356 p. Russian.

2. Dennison AR, Paraskevopoulos JA, Kerrigan DD, Shorthouse AJ. New thoughts on the aetiology of haemorrhoids and the development of non-operative methods for their management. Minerva Chir 1996; 51 : 209-216. https://www.ncbi.nlm.nih.gov/pubmed/8783861.

3. Muhin AG, Volkov AV, Komarova MY. Treating hemorrhoids in outpatient settings. Coloproctologia 2010; 31: 18-21. Russian. https://elibrary.ru/item.asp?id=15237763.

4. Laufer MD, Farley BE, inventors; Vnus Medical Technologies, Inc., assignee. Method for treating hemorrhoids. US patent 6,135,997. 2000 Oct 24

5. Nazaria MS, Maryam KH. Comparison of intrahemorrhoidal coagulation with 980 nanometer diode laser and milligan morgan hemorrhoidectomy: a randomized clinical trial. J Clin Res Gov 2015; 4(2): 1-4. http://dx.doi.org/10.13183/jcrg.v4i2.184.

6. Senchik KY, Gryaznov NA, Kireeva GS. Medical robotic systems development based on modern advances of theranostics. Robotics and Technical Cybernetics 2015; (1): 12-16. Russian. https://elibrary.ru/item.asp?id=23175712.

7. Gafton GI, Senchik KYu, Belyaev AM, Kireeva GS, Gryaznov NA, Kharlamov VV, Nikitin SA. Rationale for development of new domestic appliances theranostic apparatus for photodynamic therapy and 
fluorescent diagnostics in oncology. Voprosy Oncologii 2016; 62(5): 559-569. Russian. https://elibrary.ru/item.asp?id=27170074.

8. Giamundo P, Cecchetti W, Esercizio L, Fantino G, Geraci M, Lombezzi $R$, et al. Doppler-guided hemorrhoidal laser procedure for the treatment of symptomatic hemorrhoids: experimental background and short-term clinical results of a new mini-invasive treatment. Surg Endosc 2011; 25(5): 1369-1375. https://doi.org/10.1007/s00464-0101370-x.

\section{Authors:}

Nikolay A. Gryaznov - PhD, Deputy Director for Research, Central Research Institute for Robotics and Technical Cybernetics, Saint-Petersburg, Russia. http://orcid.org/0000-0001-5188-3585.

Vyacheslav V. Kharlamov - Head of the Research Department №6, Central Research Institute for Robotics and Technical Cybernetics, SaintPetersburg, Russia.

Sergey A. Nikitin - Head of the 604 Research Laboratory, Central Research Institute for Robotics and Technical Cybernetics, Saint-Petersburg, Russia. http://orcid.org/0000-0003-4773-0863.

Igor M. Cybin - CEO, NPF «BIOSS», Saint-Petersburg, Russia.

Konstantin Y. Senchik - MD, PhD, Senior Researcher, 604 Research Laboratory, Central Research Institute for Robotics and Technical Cybernetics, Saint-Petersburg, Russia.

Denis G. Shamarin - Programmer, 603 Research Laboratory, Central Research Institute for Robotics and Technical Cybernetics, SaintPetersburg, Russia.

Alina Y. Karseeva - Engineer, 604 Research Laboratory, Central Research Institute for Robotics and Technical Cybernetics, Saint-Petersburg, Russia.

Galina S. Kireeva - PhD, Researcher, 604 Research Laboratory, Central Research Institute for Robotics and Technical Cybernetics, SaintPetersburg, Russia; Senior researcher, Department of Carcinogenesis and Aging, N.N. Petrov Research Institute of Oncology of the Russian Ministry of Health, Saint-Petersburg, Russia. http://orcid.org/0000-0002-4732-5895. 